\title{
Making Medical Decisions for Incapacitated Patients Without Proxies: Part II
}

\author{
Eric Blackstone $^{1}$ D $\cdot$ Barbara J. Daly ${ }^{2} \cdot$ Cynthia Griggins $^{3}$
}

Published online: 6 November 2019

(c) Springer Nature B.V. 2019

\begin{abstract}
In the United States, there is no consensus about who should make decisions in acute but non-emergent situations for incapacitated patients who lack surrogates. For more than a decade, our academic medical center has utilized community volunteers from the hospital ethics committee to engage in shared decision-making with the medical providers for these patients. In order to add a different point of view and minimize conflict of interest, the volunteers are non-clinicians who are not employed by the hospital. Using case examples and interviews with the community members, this paper describes how the protocol has translated into practice over the years since its inception. Members reported comfort with the role as well as satisfaction with the thoroughness of their discussions with the medical team. They acknowledged feelings of moral uncertainty, but expressed confidence in the process. Questions raised by the experience are discussed. Overall, the protocol has provided oversight, transparency, and protection from conflict of interest to the decision-making process for this vulnerable patient population.
\end{abstract}

Keywords Unbefriended · Unrepresented · Surrogate decision-making · Substituted judgment $\cdot$ Ethics committees

\section{Background}

Acutely ill, incapacitated patients who lack a surrogate raise unique ethical challenges. Absent any clues as to the patient's preferences, who should make medical decisions? What principles should guide these decisions? These cases are

Eric Blackstone

ecb85@case.edu

1 Frances Payne Bolton School of Nursing, Case Western Reserve University, 10900 Euclid Avenue, Cleveland, OH 44106-4904, USA

2 Case Western Reserve University, 10900 Euclid Avenue, Cleveland, OH 44106-4904, USA

3 Department of Neurology, Neurological Institute, University Hospitals Cleveland Medical Center, Case Western Reserve University School of Medicine, 11100 Euclid Ave, Cleveland, $\mathrm{OH} 44106$, USA 
particularly challenging in intensive care units, where patients require complex medical management and often end-of-life decisions. For the purposes of this paper, these patients will be referred to as "patients without proxies" (PWPs), though other names such as "unbefriended" or "unrepresented" have been used in the literature (Sequeira and Lewis 2017; Courtwright et al. 2017).

In the United States, laws vary widely on who has the authority to make medical decisions for PWPs and what types of decisions they can make. While consent can be assumed in a true emergency, many non-emergent situations still require decisions earlier than the weeks-to-months it would take to appoint a guardian. A recent legal review by Thaddeus Pope highlights the confusing patchwork of laws that various states have passed in order to address this issue (2017). A number of states have taken preventative measures, such as expanding the list of legally acceptable surrogates to include more distant relatives, or friends. A few have authorized the physician to make certain decisions, often after seeking a second medical opinion. Others have authorized physicians to make decisions after consulting an ethics committee. Many limit the decisions or prescribe a "tiered" approach. Currently, there is no consistency in the law, and many states (such as ours) have yet to address the issue at all.

Individual institutions have likely developed their own procedures for PWPs, but their protocols are not widely disseminated and their outcomes are understudied. We suspect that in most cases, the medical team makes decisions for PWPs based on the best interest standard, which is supported by the findings of White et al. $(2006,2007)$. However, increasing attention to this problem in the last decade has led to the development of alternative protocols (Kim and Song 2018). In 2005, our urban hospital designed a protocol, described below, in which an ethics subcommittee composed of community volunteers meets with the medical team to make shared decisions for these patients (Hyun et al. 2006). Since then, the ethics service has received 191 referrals for PWPs and conducted 80 PWP committee meetings (Griggins et al. 2019).

The purpose of this paper is to complement the data reported by (Griggins et al. 2019) by providing a qualitative, retrospective look at the process of decision-making for PWPs. Using case examples and excerpts from semi-structured interviews with 12 committee members, we will discuss how the protocol has translated into practice. Further, we will explore the ethics and implications for future use of the protocol given our experience.

\section{PWP Protocol}

The design and implementation of our protocol has been described previously (Hyun et al. 2006). Briefly, when the medical team identifies a PWP who will require imminent, but non-emergent medical decisions, they are advised to contact the ethics consultation service. The consultant first works with the medical team and social worker to conduct an aggressive search for a surrogate, or, failing that, to find someone who knows the patient and can provide information as to what treatment the patient might have wanted. By using creative search techniques, the consultants are often successful in finding a surrogate or another source of information such as a friend, clergy, or 
previous health care provider. Occasionally surrogates are incapacitated themselves, or refuse to take on the role of decision-maker. Our state law prevents many of these people (friends, neighbors, etc.) from serving as surrogates, but they are often available and willing to contribute by telling us what they know of the patient's life and values.

If no legally acceptable surrogate can be located, the ethics consultant contacts the PWP committee members to recruit 2-3 individuals who can meet with representatives from the medical team within $24-48 \mathrm{~h}$. This meeting is facilitated by the ethics consultant, and friends of the patient (who are ineligible to serve as surrogates) are encouraged to attend and add their perspective. At the meeting, the medical team presents the medical facts of the case, the available treatment options, and their recommendations (if any). The ethics consultant and social worker provide information that was gathered about the patient's background. If available, friends share information regarding the patient's personality, values, and lifestyle, and give input as to what they think the patient might have wanted in this situation. PWP committee members synthesize this information, discuss, and make a recommendation that is in the patient's best interest, and consistent with his or her values (if known).

\section{Case Examples}

The following cases illustrate how the PWP protocol translates into practice.

\section{Case 1: Ms. M}

Ms. M was a 72-year-old single woman admitted with a severe subarachnoid hemorrhage. She was taken to surgery emergently to clip a cerebral aneurysm. Three days post-surgery, Ms. M experienced vasospasms and bilateral strokes. She was unconscious in the Neurosurgical ICU (NSU) when the medical team called the ethics service. The ethics consultant and social worker searched to find the patient's family. Colleagues from Ms. M's former workplace said the patient had emigrated from a foreign country decades ago. They described her as a private and fiercely independent woman, who had been active in her church. The pastor thought there might be relatives in her native country but did not know how to contact them. He confirmed that Ms. M valued her independence and had often talked about her love of travel. While she had never spoken of her wishes regarding health care at the end of life, he thought that Ms. M would not want to extend her life if she were to be severely cognitively and physically disabled.

Two members of the PWP committee met with the NSU team and social worker on two separate occasions. Initially, the medical team could not offer a prognosis for recovery, and a decision was made to continue aggressive life support, including ventilation and artificial nutrition/hydration. The consensus was to attempt extubation if Ms. M's condition improved, but not to attempt resuscitation if she suffered cardiac arrest. However, after 10 days, she still could not be weaned from the ventilator, and had shown no signs of cognitive recovery. The PWP committee met again and recommended against tracheostomy and PEG placement, instead opting 
for comfort care. They based this decision on the belief that an active, independent woman like Ms. M would most likely not want an extended life of disability in a nursing facility. The attending physician agreed. Ms. M was extubated and died $4 \mathrm{~h}$ later.

\section{Case 2: Ms. F}

Ms. F was a 57-year-old homeless woman with schizophrenia and mild cognitive impairment. She had fallen and suffered a hairline fracture of her ankle. In the emergency room, she had been fitted with a boot and crutches, told not to put weight on the ankle, and to follow up with orthopedics. Ms. F failed to follow through with the orthopedic appointment, and had been walking as usual. When she returned to the emergency room, she had fallen again, and now required surgery and implanted hardware to repair the ankle. She refused the surgery, saying she would "wear the boot and use the crutches this time." However, the medical team believed that she lacked capacity to make this decision, and called ethics. Ms. F had long ago alienated her family, so none could be located. Neither the mental health center where she had received treatment intermittently, nor the local police, who knew Ms. F well, was aware of any relatives. Search of the county Probate court records showed that no guardian had ever been appointed for her.

The PWP committee met with members of the medical team, social work, and psychiatry on day 2 of the hospitalization. They learned that she was stable from a psychiatric perspective, and without surgery, it would be impossible for Ms. F's ankle to heal and for her to be ambulatory again. Given that she was in relatively good health, complications were not anticipated. However, Ms. F would likely require extended time for recovery, and placement in a nursing facility following discharge was recommended. The PWP committee met Ms. F and tried to understand her reasons for refusing surgery, but she could not articulate any. The PWP committee deliberated and agreed with the medical team's recommendation of surgery, casting, and discharge to a nursing facility. They also recommended that a guardian be found for her. Ms. F eventually assented to surgery and was subsequently discharged to a nursing facility for rehabilitation.

\section{Case 3: Mr. G}

Mr. G was a 69-year-old man admitted for anemia and found to have colon cancer. The source of his incapacity was not entirely clear, though the medical team felt he had baseline dementia and may have had a stroke in the past. Three members of the PWP committee met with the colorectal surgeon, clinical ethicist, and Mr. $\mathrm{G}$ to discuss surgery. The surgeon explained the details and risks of a hemicolectomy, including the possibility of ileostomy. Mr. G insisted that he wanted surgery but "I don't want a bag!" He also kept repeating that he wanted to get back to his home. Committee members questioned the surgeon about the nature of his recovery, and whether any alternative options were available. The only alternative would be comfort care, but the surgeon feared eventual bowel obstruction, which would cause 
significant discomfort. She thought Mr. G was a good candidate for surgery and anticipated a positive outcome. The need for an ileostomy was unlikely, but possible, and potentially reversible. Mr. G. could not understand that his desire for surgery carried the necessary risk of ileostomy. Given that both of his wishes could not be honored, the committee weighed the benefits and risks, and consented for surgery.

The committee met a second time in the Surgical ICU, after Mr. G had undergone a second emergent surgery, necessitating an ileostomy. Mr. G was now intubated, with fluid collecting in his abdomen. The committee consented for the placement of drains and various diagnostic tests, but recommended that a "Do Not Attempt Resuscitation" (DNAR) order be instituted after he was stable enough to be extubated. They also recommended that an application for guardianship be started, anticipating a prolonged hospital stay and recovery.

Mr. G was able to leave intensive care, but he was refusing to eat or get out of bed. After weeks of poor intake, Mr. G became increasingly frail and deconditioned. The committee was asked to consent for feeding tube placement and discharge to a nursing facility. However, given Mr. G's repeated desire to return home, which was now extremely unlikely, given his steady decline, the committee recommended hospice. A guardian was finally appointed by the court, who agreed to enroll Mr. G in hospice care.

\section{Interview Methods}

The protocol utilizes community volunteers who are members of the hospital ethics committee. A semi-structured interview was approved by the Institutional Review Board and all 12 PWP committee members consented to participate. In addition to providing demographic information, interviewees answered open-ended questions relating to several core aspects of the PWP protocol, which can be found in Box 1.

\section{Box 1: Content of Structured Interview with PWP Committee Members}

- Demographic questions (age, sex, religion, education, occupation)

- Personal experience in healthcare decision-making (for self or family members)

- Member's assessment of personal qualifications required for committee membership and preparation for role

- Member's experience with PWP cases (mechanics, medical and social information provided, quality of discussion, meeting the patient)

- Member's views on how personal values and religious beliefs affect their decision-making

- Member's experience and views on the post-decision period (second-guessing, need for feedback, debriefing) 


\section{Committee Demographics}

Demographics of the PWP committee members are shown in Table 1. Because the purpose is not to second-guess the medical facts, the members are intentionally not medical practitioners, and in order to minimize conflict of interest, they cannot be hospital employees. Although no educational requirements are specified in the protocol, because the members are recruited by the hospital's ethics consultants, most of the volunteers have some education in bioethics in addition to their experience on the ethics committee. All but one are college graduates with post-graduate degrees. All have clinical experience from varied perspectives-clinical research, clinical rotations while in school, pastoral care, nursing home advocacy, or personal experience as a patient or caregiver. At the time of the interviews, their experience with PWP cases ranged from 2 to 18 cases over 1 to 10 years of participation in the committee.

\section{Committee Member Characteristics}

\section{Qualifications and Training}

There are no specific qualifications for serving as a PWP except for the requirement that the individual be an active member of the hospital ethics committee in order to gain experience with clinical situations, ethical dilemmas, and sound decision-making processes. Membership on the hospital ethics committee is also required so that members are formally registered as hospital volunteers. Volunteers receive HIPAA training, authorization to have access to patients' private health information, and protection from liability. In addition, it is important that members have basic knowledge of clinical terms and common medical procedures, as the medical team can vary in their patience and ability to communicate effectively with laypersons. It is also important that committee members are available at short notice. Decisions are usually needed urgently, so members are asked to meet with the medical team within 24-48 h after receiving the consult. It has been helpful to have committee members who live or work close to the hospital and are retired or have flexible schedules.

Over the years since the inception of the protocol, committee orientation and education have become somewhat more formalized. Three of the original members did not recall any formal training beyond participating in the larger ethics committee. More recent recruits were asked to meet with the ethics consultants, read and discuss an article about the PWP protocol, and sit in on PWP consults as an observer. This combination of clinical experience, serving on the ethics committee, and observing PWP cases seems to be adequate, as they are not expected to provide expert ethical 
Table 1 PWP committee member demographics

\begin{tabular}{lc}
\hline Characteristic & $\mathrm{N}$ \\
\hline Gender & \\
Male & 5 \\
Female & 7 \\
Age (years) & \\
$25-40$ & 4 \\
$41-55$ & 3 \\
$56-70$ & 4 \\
$>71$ & 1 \\
Race & \\
White & 11 \\
Asian & 1 \\
Religion & \\
Catholic & 2 \\
Jewish & 2 \\
Protestant & 1 \\
None & 5 \\
Refused & 2
\end{tabular}

Highest education level

Master's

$\mathrm{PhD}$

2

JD

3

HS diploma 1

Discipline

Bioethics 5

Law 3

Other (nursing, rabbinical studies, clinical research) $\quad 4$

Occupation

Research assistant 2

Professor/instructor 6

Medical writer 1

Rabbi 1

Musician 1

Homemaker 1

Training in bioethics

$\mathrm{PhD}$

M.A. $\quad 3$

Certificate 1

None 6 
opinions. Rather, they are serving in place of the laypersons (family proxies) who would, if available, be making decisions for these patients.

Regarding their qualifications, when interviewed, all members felt that they were at least "mostly" qualified to serve on the PWP committee. When asked what qualifications they felt were necessary, there was a consensus that training in ethics and clinical experience were desirable. For example, members stated:

- "Some medical understanding ... knowing how and when to ask questions of the medical team."

- "A realistic view of what medicine can and cannot do."

- "Familiarity with how hospitals work...knowledge of the standards of decisionmaking...."

Committee members also listed other, less tangible qualities that help with PWP decision-making, such as communication skills, compassion, empathy, and the ability to cope with ambiguity. Communication skills are vital for interacting with one another as well as the medical team. PWP meetings involve people with varying backgrounds and areas of expertise, so members must be able to appreciate other perspectives and not be reticent about raising their own questions.

Committee members must also be able to cope with the doubt and ambivalence inherent in these decisions. Although some background information on patients often can be found, the committee is usually working with an incomplete picture of who the patient is and what that person would have wanted. Knowing only fragments of the patient's personality and values, they must make difficult decisions about treatments that can have serious risks and uncertain benefits. In addition, there is a specific discomfort in knowing that someone else must bear the consequences of one's decision. As one committee member put it, "the ability to appreciate ambiguity and live with some uncertainty" is a requirement.

\section{Personal Values and Experience}

A concern in any type of surrogate decision-making is that one's personal values and biases may cloud judgment and interfere with determining the best interest of the patients. When asked how their personal values, philosophy, or religious beliefs affected their decisions for PWPs, committee members acknowledged that they were influential despite their attempts to remain objective:

- "Individual values always underlie these kinds of processes."

- "I have difficulty knowing if that person would share my philosophy. There is a vacuum since the patient cannot tell you what his or her values are."

- "I know I would want everything done, except if there is irreversible suffering." 
- "I lean towards comfort care-I know I do... [but] I don't think it makes me too quick to shut down other choices. I worry so much that could happen that I go overboard to look at the other choices."

The lack of information about PWPs likely leads to some projection of committee members' values onto patients. The quote above mentions a vacuum-the patient's own values are absent and must be assumed. There is no coherent way to make a decision without filling this void. This is why substituted judgment by family is the preferred standard of decision-making - they have the most information about the patient and are instructed to follow the patient's wishes and values. They are therefore less susceptible to bias. However, even those who know the patient best are not perfectly accurate and do not always utilize substituted judgment. Ditto and colleagues found that family surrogates could predict the patient's wishes with 66-82\% accuracy depending on the type of decision (2001), leaving 18-34\% of decisions not in agreement with what the patient would want. Further research demonstrated that family predictions about the patient's treatment preferences more closely match their own (Fagerlin et al. 2001). Given that the PWP committee has limited information about the patient's life and values, there is even more concern that personal values and biases may seep into the decision-making process.

It was notable, however, that when committee members with religious affiliations were interviewed, they made no comment about the effect of their beliefs on their decision-making process. There are several possible explanations for this. They may not be aware or comfortable sharing that their religious framework affected their decisions, or they may try to put their religious beliefs aside, accepting that the patient may not share those beliefs.

Most interviewees had personal experiences with making medical decisions for family members that influenced their decision-making for PWPs:

- "[I made decisions for] an 80 year-old aunt with advanced dementia... the family had to work with the team on withdrawing nutrition and hydration. Consequently, I always have the recognition that I am not just dealing with a patient in a bed, but a real person with real history, regrets, conflicts, love, goals, etc."

- "My son-in-law had colon cancer... took every treatment and fought "til the end. I knew he wasn't going to make it."

- "My own medical condition frequently put me in the hospital and gave me perspectives I otherwise wouldn't have."

The PWP committee members felt that these experiences made them more comfortable being direct with the doctors, and gave them a clearer understanding of comfort care and hospice. We do not know if these experiences lead to better (or worse) decision-making for PWPs, but it seems plausible that familiarity with making decisions for another would be advantageous. Feeling the responsibility of endof-life decision-making and seeing how those decisions affected their family member may provide valuable insight. On the other hand, over-generalizing personal experiences may be detrimental to the decision-making process. A family member may have had a beneficial or a poor outcome with a certain treatment, but that is not 
necessarily representative of all patients and may lead to bias. The PWP decisionmaking protocol relies on consensus among multiple committee members and the medical team in the hopes that differing perspectives and experiences will neutralize individual biases.

\section{Decision-Making Process}

\section{Meeting Quality}

When asked about their experience in meeting with the medical providers, committee members, for the most part, felt satisfied with the meetings and discussion. They occasionally felt rushed if the medical team had limited time, but generally, they felt the discussions were thorough and their input was taken seriously. Surprisingly, the medical team did not always offer a clear recommendation or opinion. Committee members perceived that physicians appreciated the diffusion of responsibility for these decisions, freeing them to focus on the medical management of the patient. One committee member supposed that, "[The medical team] wants input because it takes some of the responsibility off their shoulders."

The committee members almost universally felt comfortable asking the medical team questions during meetings. All but one interviewee felt that they received adequate medical information about the patient during their meetings with the medical team. However, many commented that accessing the necessary information required assertive questioning on their part. Several committee members also expressed concern that various members of the medical team gave them inconsistent or conflicting information. Possibly mirroring the experience of family surrogates, two members commented:

- “...sometimes you really have to get it out of them... regarding likelihood of success, they can be wishy-washy."

- "Sometimes you get conflicting opinions from different doctors; one says the patient is improving and the other says "no way,' that is very disconcerting."

Committee members were satisfied with the efforts of the ethics consultant and social worker to gather information about the patients and saw this as an important part of the process. The ethics consultants were viewed as mostly impartial and helpful in guiding the discussion. Committee members did not feel pressured by them, despite the consultants occasionally making their opinions known.

One potential threat to the quality of these meetings is the phenomenon of groupthink, a bias that results from a cohesive group trying to reduce the stress of decision-making by suppressing critical thinking (Janis 1972). However, when asked, committee members did not seem to view groupthink as a serious threat to the decision-making process. They expressed confidence in themselves and the other members in terms of their ability to think independently and raise alternative points of view. 
It may be that the members' confidence is related to the size of the typical PWP meeting. Research about group decision-making suggests that group task performance increases with group size until it reaches five members (Treen et al. 2016). PWP meetings generally have 5-6 people present: an ethicist, a social worker, 1-2 members of the medical team, and 2 committee members. Thus, the PWP meetings seem to have the right group size to gain multiple perspectives while minimizing groupthink. Of course, group size and the fact that committee members did not express concern about it do not rule out groupthink entirely. It is certainly possible that the committee recruited members who already tend to agree with one another. All recruits serve on the full ethics committee and therefore may have absorbed the culture or attitudes of the broader group. Members may also be unaware or unwilling to admit that they succumbed to this bias.

\section{Key Factors in Decision-Making}

When asked what type of information was most influential when making decisions, committee members mentioned prognosis, quality of life, minimizing suffering, the risks and benefits of proposed interventions, and the patient's wishes if known.

- "It varies based on the decision; if terminal illness, then quality of life, and if treatment, then the risks and benefits of the options."

- "Prognosis and the potential for suffering without gain."

- "What will be their quality of life when it's all over, and what's recuperation like? How much pain? I didn't want to see anyone linger in pain. This is the one thing we can do for people at the end-prevent unnecessary suffering."

- "Age does factor in if chances are not great and if dealing with a 90-year-old versus a 50-year-old. It's not purely ageism-it depends on the patient's condition and perceived ability to experience benefit and achieve desirable goals."

Committee members also commented on the need to look past the current treatment decision to anticipate what recovery would entail. This is a unique challenge in decision-making for PWPs. Social support has long been recognized as a predictor of recovery (Prang et al. 2015), but given their isolation, PWPs generally will not have someone to assist them and encourage adherence to recommendations after discharge. Ms. F's case is an example of the importance of this factor; in the absence of family or health system support, she was unable to comply with follow-up and continued to put weight on her ankle despite the medical recommendations.

\section{Patient Involvement}

The question of involving incapacitated patients in the decision-making process has been an ongoing issue for the committee members. Although by definition all PWPs lack capacity to make medical decisions, their mental status can range from coma to completely awake and alert. This has challenged the committee to determine how best to involve the patients. Should unconscious patients at least be visited 
by the committee? Should alert, but cognitively impaired patients be interviewed by the committee, or even allowed to attend the formal PWP meetings and voice their (incapacitated) opinions?

The discussion over visiting comatose or obtunded patients was sparked by a committee member who felt that lay people could be biased by seeing critically ill patients. Nonprofessionals unused to seeing sick and dying patients may be affected in unpredictable ways, lacking the medical knowledge to contextualize what they are seeing (Robichaud 2015). Interviewees expressed a variety of opinions about this, weighing the benefits of gaining additional information and humanizing the patient against the risk of introducing bias into the decision-making process. Ultimately, most committee members felt that seeing the patient was at the very least permissible and in some cases obligatory. We agree that seeing critically ill patients may elicit a strong emotional response from committee members, and that medical professionals are much less likely to react in such a way. It is the role of the medical team to provide context for what the committee members are seeing. Their differing perspectives complement one another; the clinicians bring their own biases that laypersons do not, and vice versa. This is the strength of the PWP protocol - bringing together physicians, nurses, social workers, ethicists, and community members so that each may add a unique perspective and counterbalance the biases of others.

PWPs who are alert, awake, and voicing their (incapacitated) opinions are perhaps the most challenging. They often express inconsistent or conflicting wishes that are impossible to honor. In the case of Ms. F, the necessary treatment and prognosis were clear enough that the committee overruled her expressed wish to refuse surgery and "wear the boot." However, in more complex and nuanced situations, hearing a patient's poorly articulated or conflicting opinions can be problematic. Committee members often reminisce about Mr. G because his case encapsulated the ambivalence and discomfort inherent in listening to an incapacitated patient. His opinions and preferences changed daily and he was unable to explain the bases of his decisions. This made it difficult to support his wishes, yet caused some guilt and discomfort when going against them.

\section{Discussion}

The PWP protocol has been successful in several ways. It represents a feasible solution to a long-standing problem of who will make decisions for incapacitated patients when there is no surrogate. It is consistent with Pope's recommendations for a process that is "....accessible, convenient, and cost-effective... that provides the important safeguards of expertise, neutrality, and careful deliberation" (2017, p. 931). Though we did not interview clinicians to gain their perspective on the proto$\mathrm{col}$, it seems to be well accepted as evidenced by the steadily increasing number of referrals-from 6 cases in 2006 to 42 in 2016 (Griggins et al. 2019). Feedback from the committee members indicates their satisfaction as well, but also brings to light questions and issues about the process.

The most important consideration is whether the committee members, in conjunction with the medical providers, are making the "right" decisions, or at least 
ethically defensible decisions. As it is impossible to answer the former question, we can only address the latter by considering the goal of the process and the necessary components of ethically defensible decision-making for these patients.

It is debatable whether the goal of the committee is to honor the patient's autonomy by ascertaining, as much as possible, what the patient might have chosen (substituted judgment), or to determine what is in the patient's best interest. Substituted judgment and best interest standards of decision-making are often presented as mutually exclusive, but PWP decision-making according to our protocol involves elements of both. Depending on how much background information the ethicist and social worker can discover, it is sometimes possible to make a reasonable guess as to what the patient would have wanted. Perhaps a neighbor says the patient was fiercely independent and valued physical activity above all other aspects of life; or staff at the patient's nursing home describe a patient's recognition of poor quality of life and expressed wishes to stop treatment. In these cases, there is at least some indication that a patient's wish might be to set limits on treatments, or forego them entirely. Alternatively, knowing that a patient had a positive outlook and adjusted well to life in a nursing facility does not tell us exactly what a patient would want, but suggests the patient's tolerance for extended rehabilitation or permanent residence in a facility. Piecing together these bits of biographical information does not suggest that substituted judgment in the classic sense is being exercised. However, it does go beyond deciding what is in the "best interest" of a generic and completely unknown patient, and presumably moves in the direction of supporting patient autonomy.

Ethical decisions can only be achieved if the appropriate individuals are involved, and there has been some debate in the literature about who should be making decisions for patients who lack proxies: physicians or third parties such as ethics committees. Courtwright and Rubin argue that physicians should make these decisions, not just because they are most knowledgeable about the patient's condition and treatment options, but because the physician's fiduciary duty to the patient compels him/ her to act as the surrogate (2015). Alternatively, White et al. argue that physicians' expertise in medicine does not extend to the "complex social, ethical, and legal considerations" inherent to PWP decision-making (2012). In addition, physicians may have conflicts of interest caused by institutional or financial pressures. They may be subject to medical bias by considering only medical facts and adhering exclusively to decisions guided by evidence-based medicine. Instead, White recommends that decision-making authority be given to "someone not encumbered by relationships with the institution and the clinicians involved in the case." Our protocol recognizes both viewpoints by relying on a consensus of both the medical providers and nonmedical committee members.

Just as shared decision-making is the preferred model when surrogates are available (Elwyn et al. 2012), we believe that shared decision-making is appropriate for patients without surrogates. The individuals who dialogue with the physicians should be as free of bias as possible, yet knowledgeable in ethical decision-making, and offer additional perspectives to those of the medical providers. Our protocol recognizes the fiduciary responsibility of the physician in that he/she retains ultimate authority for providing medical treatments. However, it is notable that there 
have been no cases in which the committee and the physician were unable to reach consensus.

The question remains, however, whether the committee members are truly bringing unbiased and diverse perspectives to the deliberations. While they are members of the community, the committee is not demographically representative of the patients for whom they make decisions. There is a good balance of age and gender, but they are more educated than the general population, economically more well off, and do not reflect the racial diversity of our PWPs- $51 \%$ of whom are African American (Griggins et al. 2019). We hypothesize that educational differences between the committee members and the PWPs might not be problematic, but in fact might be advantageous. Committee members have higher levels of health literacy and clinical experience in decision-making than most patients or surrogates, which we believe enable them to ask more probing questions and even disagree with the medical team at times. One can imagine that traditional surrogates might lack the knowledge or confidence to challenge medical professionals. However, the lack of racial diversity is a significant weakness. A more racially diverse committee could offer cultural knowledge and experience missing from the committee at present. Likewise, recruiting more individuals who have experienced disability and serious illness would also add valuable perspectives.

In addition to having the right people making the decisions, the process by which they come to those decisions must also be ethically sound. With limited knowledge of patients and their wishes regarding treatments, decision-making based on substituted judgment is not possible. However, we feel that the protocol facilitates decision-making that is ethically more desirable than "best interest" based on what a "reasonable person" would want given the risks, benefits, and likelihood of success. Because we have been able to gather important information about the patient's lifestyle and quality of life in almost all cases, we believe that we are utilizing a hybrid standard, which we have termed "informed best interest". We believe that "informed best interest" is best determined by rigorous dialogue between the medical team and committee members. Committee members do not perceive they are simply "rubber-stamping" the doctor's decision; rather, they have thorough discussions commensurate with the gravity of the decision at hand. The process is made transparent by recording all attempts to locate surrogates and information gathered about each patient, as well as the deliberations and decisions made for them. Finally, fairness is served by adherence to the same protocol for every PWP case.

For the reasons stated above, we feel that our protocol is making the best of what will always be a non-ideal situation. Our protocol ensures that every attempt is made to respect the autonomy of these patients by mandating a thorough search for suitable surrogates and other evidence of the patient's wishes. It minimizes medical and institutional bias by including community members in the decision-making process. By identifying these patients, aggressively searching for family and background information, reaching a consensus on the most ethical treatment plan, and tracking outcomes, we have established a level of transparency and oversight that was previously lacking.

That said, the input from the volunteers serving in this important role suggests that the process could be improved in several ways. Providing treatment 
recommendations that can be life altering, and in some cases, life ending, can generate feelings of discomfort and moral ambiguity. Several interviewees admitted to occasionally dwelling on past cases and wondering whether they made the right decision. A more formalized review and debriefing process could be initiated, which would be helpful for quality control and training, as well as a source of closure for the committee members. At this time, PWP cases are reviewed on a regular basis by the full ethics committee. The purpose is to ensure rigor and consistency in the decision-making process, as well as to inform committee members about the outcomes of their cases. However, it would be beneficial to also debrief committee members periodically, one-on-one, to provide an opportunity to discuss any concerns they may not be comfortable sharing with the full committee. Talking through challenging cases with others may lessen any negative feelings and protect against burnout. While these negative feelings are an important concern, the committee members also take great satisfaction in helping these patients. It is worth noting that no PWP committee members have dropped out or avoided meetings due to burnout.

\section{Conclusion}

For most hospitals in the United States, the process for making non-emergent decisions for PWPs has not been widely reported or studied. At the very least, our protocol identifies these patients and provides transparency and accountability in decision-making. We have a committee of community members who are empathic and knowledgeable in clinical and ethical decision-making, doing their best to represent patients who otherwise have no one to speak for them. Their background and experience give them the confidence to question the medical team thoroughly, understand the options, and make a reasoned judgment based on "informed best interest". No protocol for PWP decision-making will avoid the moral discomfort and uncertainty involved in making decisions for this patient population, nor should that be the goal. Rather, PWP protocols should focus on improving on the status quo by implementing thorough procedures for finding background information about the patient, promoting transparency, and minimizing conflict of interest.

Acknowledgements Olubukunola Dwyer and Lauren McAliley for assisting with the interviews, and the PWP committee volunteers.

\section{Compliance with Ethical Standards}

Conflict of interest The authors have no conflict of interest to disclose.

\section{References}

Courtwright, A. M., Abrams, J., \& Robinson, E. M. (2017). The role of a hospital ethics consultation service in decision-making for unrepresented patients. Journal of Bioethical Inquiry, 14(2), 241-250.

Courtwright, A., \& Rubin, E. (2015). Who should decide for the unrepresented? Bioethics, 30(3), $173-180$. 
Ditto, P. H., Danks, J. H., Smucker, W. D., Bookwala, J., Coppola, K. M., Dresser, R., et al. (2001). Advance directives as acts of communication: A randomized controlled trial. Archives of Internal Medicine, 161(3), 421-430.

Elwyn, G., Frosch, D., Thomson, R., Joseph-Williams, N., Lloyd, A., Kinnersley, P., et al. (2012). Shared decision making: A model for clinical practice. Journal of General Internal Medicine, 27(10), 1361-1367.

Fagerlin, A., Ditto, P. H., Danks, J. H., Houts, R. M., \& Smucker, W. D. (2001). Projection in surrogate decisions about life-sustaining medical treatments. Health Psychology, 20(3), 166-175.

Griggins, C., Blackstone, E., McAliley, L. et al. (2019). Making medical decisions for incapacitated patients without proxies: Part I. HEC Forum. https://doi.org/10.1007/s10730-019-09387-3.

Hyun, I., Griggins, C., Weiss, M., Robbins, D., Robichaud, A., \& Daly, B. (2006). When patients do not have a proxy: A procedure for medical decision making when there is no one to speak for the patient. Journal of Clinical Ethics, 17(4), 323-330.

Janis, I. L. (1972). Victims of groupthink. Boston: Houghton Mifflin.

Kim, H., \& Song, M. (2018). Medical decision-making for adults who lack decision-making capacity and a surrogate: State of the science. American Journal of Hospice and Palliative Medicine, 35(9), 1227-1234.

Pope, T. M. (2017). Unbefriended and unrepresented: Better medical decision making for incapacitated patients without healthcare surrogates. Georgia State University Law Review, 33(4), 923-1019.

Prang, K. H., Berecki-Gisolf, J., \& Newnam, S. (2015). Recovery from musculoskeletal injury: The role of social support following a transport accident. Health and Quality of Life Outcomes, 13, 97.

Robichaud, A. L. (2015). Medical decision making for patients without proxies: The effect of personal experience in the deliberative process. Journal of Clinical Ethics, 26(4), 355-360.

Sequeira, A., \& Lewis, A. (2017). Ethical and legal considerations in the management of an unbefriended patient in a vegetative state. Neurocritical Care, 27(2), 173-179.

Treen, E., Atanasova, C., Pitt, L., \& Johnson, M. (2016). Evidence from a large sample on the effects of group size and decision-making time on performance in a marketing simulation game. Journal of Marketing Education, 38(2), 130-137.

White, D. B., Curtis, J. R., Lo, B., \& Luce, J. M. (2006). Decisions to limit life-sustaining treatment for critically ill patients who lack both decision-making capacity and surrogate decision-makers. Critical Care Medicine, 34(8), 2053-2059.

White, D. B., Curtis, J. R., Wolf, L. E., Prendergast, T. J., Taichman, D. B., Kuniyoshi, G., et al. (2007). Life support for patients without a surrogate decision maker: Who decides? Annals of Internal Medicine, 147(1), 34-40.

White, D. B., Johnsen, A., \& Lo, B. (2012). Ethical challenge: When clinicians act as surrogates for unrepresented patients. American Journal of Critical Care, 21(3), 202-207.

Publisher's Note Springer Nature remains neutral with regard to jurisdictional claims in published maps and institutional affiliations. 